\title{
Rotation of a strongly coupled dust cluster in plasma by the torque of an electron beam
}

\author{
D. Ticoş $\odot,{ }^{1}$ A. Scurtu $\odot,{ }^{1}$ J. D. Williams, ${ }^{2}$ L. Scott $\odot,{ }^{3}$ E. Thomas Jr. $\odot,{ }^{3}$ D. Sanford,${ }^{4}$ and C. M. Ticoş $\oplus^{1,5,{ }^{*}}$ \\ ${ }^{1}$ National Institute for Laser, Plasma and Radiation Physics, Măgurele 077125, Romania \\ ${ }^{2}$ Department of Physics, Wittenberg University, Springfield, Ohio 45501, USA \\ ${ }^{3}$ Department of Physics, Auburn University, Auburn, Alabama 36849, USA \\ ${ }^{4}$ Department of Physics, Baylor University, Waco, Texas 76706, USA \\ ${ }^{5}$ Extreme Light Infrastructure-Nuclear Physics (ELI-NP), Horia Hulubei National Institute for Physics \\ and Nuclear Engineering, Măgurele 077125, Romania
}

(Received 3 May 2020; revised 18 November 2020; accepted 29 January 2021; published 17 February 2021)

\begin{abstract}
A 1-mm-size cluster composed of 10 dust particles immersed in plasma is rotated by the torque of a pulsed electron beam with energy in the range 8-12 keV. The dust particles are electrically charged spheres with radius $5.9 \mu \mathrm{m}$ and are levitated in the plasma sheath, forming a round, planar, Coulomb-coupled cluster. The electron beam irradiates the dust cluster passing slightly off its center, and sets the particles in motion by the action of the electron drag force. The total torque at $12 \mathrm{keV}$ is $4.9 \pm 0.2 \times 10^{-11} \mathrm{Nm}$ at an angular speed $1.41 \pm 0.05 \mathrm{rad} \mathrm{s}^{-1}$. The main dynamical features of the cluster such as intershell rotation and itinerancy of the dust particles inside the cluster are simulated by using a molecular dynamics code.
\end{abstract}

DOI: 10.1103/PhysRevE.103.023210

\section{INTRODUCTION}

Since the discovery that radiation pressure can move small physical objects [1] many applications have emerged in physics, nanosciences and biology where atoms, nanostructures, bacteria, and living cells are manipulated with lasers [2-5]. Aside from a photon flux, an electron beam (EB) with sufficient energy can also exert a force that is capable of displacing atoms and nanostructures [6-8]. It has been shown that even large microscopic objects such as dust particles levitated in plasma can also be moved by the drag force of a collimated electron beam [9-12]

Dust particles immersed in plasma acquire electrical charge on their surface and form regular spatial structures (also called crystals) when they become strongly coupled [13-15]. External perturbations such as a focused laser beam [16-19], a magnetic field [20-25], an electric field [26] or a neutral gas flow $[27,28]$ can well be used to set in motion these dust particles. The rotation of strongly coupled clusters and crystals in plasma has been associated with the application of different driving forces. In experiments with magnetic fields it has been suggested that the the Lorenz force of the flowing ions exerts a toroidal drag [20,22,25]. In experiments with lasers the photon pressure was the drive behind the rotation [16-19]. Rotation of a number of crystal layers has been demonstrated using the drag force of two couterpropagating lasers producing a well localized torque on the crystal [19]. A similar rotation mechanism has also been observed in strongly

\footnotetext{
*catalin.ticos@eli-np.ro
}

Published by the American Physical Society under the terms of the Creative Commons Attribution 4.0 International license. Further distribution of this work must maintain attribution to the author(s) and the published article's title, journal citation, and DOI. coupled ion crystals by applying a controllable torque from a laser beam in combination with a rotating electric field [29].

In this report we demonstrate that a collimated pulsed EB with energy in the range $8-12 \mathrm{keV}$ can rotate a small strongly coupled dust cluster in plasma. The torque is produced by the momentum transfer from the beam electrons to the dust particles. Molecular dynamics simulations of the irradiated dust cluster confirm the experimental findings.

\section{EXPERIMENT}

The cluster is composed of 10 identical spherical dust particles with radius $r_{d}=5.9 \mu \mathrm{m}$ and made of melamineformaldehyde with density $\rho=1.5 \mathrm{~g} \mathrm{~cm}^{-3}$. Eight of them are equally spaced and have an elliptical arrangement, while the remaining two are situated inside the ellipse [30]. The major and minor axes of the ellipse are $1.10 \times 0.99 \mathrm{~mm} \pm 0.01 \mathrm{~mm}$.

The dust cluster is immersed in the sheath of a radio-frequency (RF) plasma and levitated at a height $\approx 3.8-4.2 \mathrm{~mm}$ above the RF electrode, inside the cut of a metallic ring, as shown in Fig. 1. The diameter of the cut $(10 \mathrm{~mm})$ is comparable with the plasma sheath width $\approx 4.5-5 \mathrm{~mm}$. The plasma sheath is strongly deformed over the ring, and thus makes at the center of it a relatively "abrupt" potential well inside which the cluster resides.

The plasma is excited at $13.56 \mathrm{MHz}$ and $\mathrm{RF}$ power 3 to $4 \mathrm{~W}$. It is produced in $\mathrm{Ar}$ at $93-96 \times 10^{-3}$ Torr, between parallel plate electrodes. The electron density in the plasma is $n_{e}=1.3-3.7 \times 10^{14} \mathrm{~m}^{-3}$. The estimated charge of each dust particle is $Q_{d} \approx-14.9 \pm 3 \times 10^{3} \mathrm{e}$, where $e=1.6 \times$ $10^{-19} \mathrm{C}$ [12]. The RF plasma chamber design is such that it allows the injection of an electron beam through an orifice with diameter $0.5 \mathrm{~mm}$, drilled on a side flange [31]. The distance between the orifice and the cluster is $70 \mathrm{~mm}$. The EB is produced in a separate plasma chamber by extracting and accelerating free electrons from a hollow anode Penning discharge, and passed into the RF chamber after collimation into 


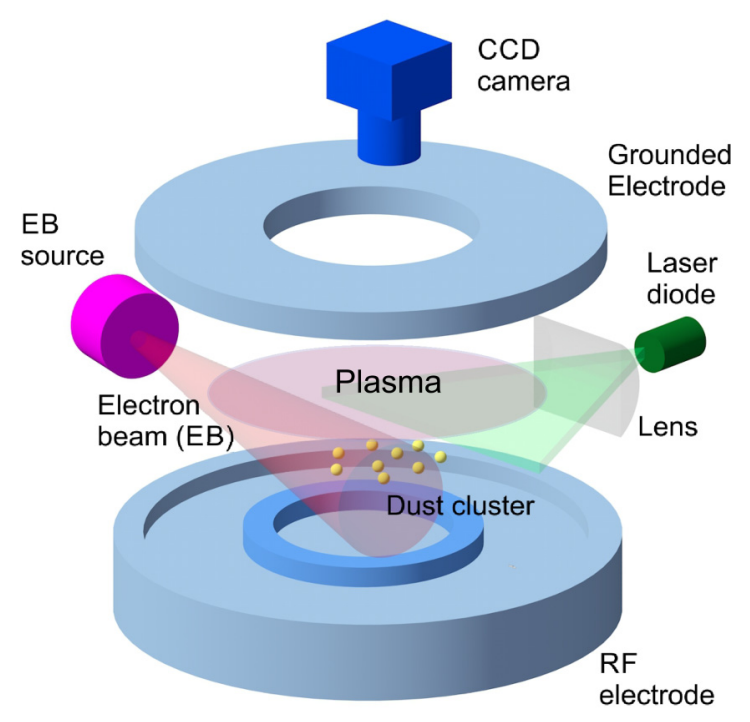

FIG. 1. A strongly coupled planar dust cluster is irradiated by an EB while it is levitated in the RF plasma sheath. A metallic ring with an inner diameter of $10 \mathrm{~mm}$ placed on the RF electrode confines the cluster to its center. A sheath of light obtained from a laser diode and a cylindrical lens is used to illuminate the cluster. The cluster dynamics is monitored from the top with a Photron CCD camera operating at $60 \mathrm{fps}$ equipped with a Micro Nikkor $40 \mathrm{~mm} \mathrm{f} / 2.8$ objective and two teleconverters $(\times 2$ and $\times 3)$.

a high vacuum pipe with axial magnetic fields of up to $150 \mathrm{G}$ produced by two circular coils [32]. The EB formed inside this high vacuum pipe (which has about $8 \mathrm{~cm}$ in diameter and $40 \mathrm{~cm}$ in length and a pressure in the range $10^{-5} \ldots 10^{-4}$ Torr) can be focused under the action of the magnetic field to a spot size $\approx 3 \mathrm{~mm}$ in diameter, and beam current in the several tens of $\mathrm{mA}$. Only a small part of this EB goes through the $0.5 \mathrm{~mm}$ orifice and is further utilized in the experiment. In order to maximize the $\mathrm{EB}$ in the RF plasma chamber, the electron beam must be focused and centered on this orifice. Since the trajectories of the electrons in a magnetic field depend on their energy, below $8 \mathrm{keV}$ the beam cannot be properly focused and only a very limited number of electrons enter into the RF chamber (corresponding to a beam current less than $0.2 \mathrm{~mA}$ ). The flange with the $0.5 \mathrm{~mm}$ orifice is necessary in order to maintain a high vacuum inside the pipe given that in the RF chamber the gas pressure is above $90 \times 10^{-3}$ Torr.

The resulting EB used to irradiate the cluster is slightly diverging reaching a diameter of $4.5 \pm 0.5 \mathrm{~mm}$ at full width at half maximum (FWHM). The EB central axis is situated at $1.3 \pm 0.2 \mathrm{~mm}$ from the cluster center. The EB current is $\approx 0.2$ to $1.2 \mathrm{~mA}$ for the range of $\mathrm{kV}$ operation, while the $\mathrm{EB}$ pulse frequency is kept constant at $56 \mathrm{~Hz}$. The pulse duration is $\approx 40 \mu$ s resulting in a duty factor $D \approx 2.2 \times 10^{-3}$ for the applied EB.

The EB ionizes the gas inside the chamber and can be easily imaged when the RF plasma is turned off, as presented in Fig. 2(a). The electron density in the beam is an order of magnitude lower than that of the RF plasma [12]. The image is obtained with a CCD Grasshopper 3 (by Point Grey, with resolution $2.3 \mathrm{MP}$ ) and a $50 \mathrm{~mm} \mathrm{f} / 2$ fixed focal length objective (model C Series - Edmund Optics). The total acquisition time of the CCD is set to $333 \mathrm{~ms}$. The axis of the EB passes

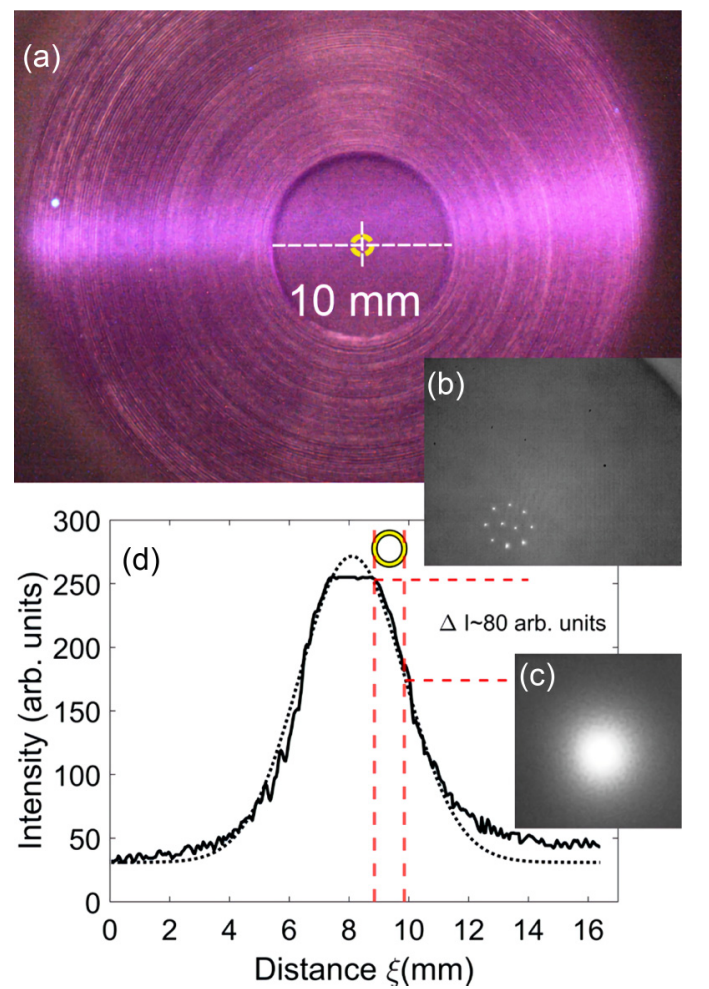

FIG. 2. (a) Top view of the pulsed EB inside $\mathrm{Ar}$ at $100 \times$ $10^{-3}$ Torr, above the RF electrode (the image has been processed by increasing its brightness). The cluster (indicated with a small yellow circle) is positioned at the center (marked with a cross) of the circular cut in the ring, near the EB axis. The diameter of the confining region is $10 \mathrm{~mm}$, while the diameter of the cluster is $\approx 1 \mathrm{~mm}$. The EB central axis is slightly displaced relative to the center of the dust cluster; (b) Top view of the dust cluster levitated in the plasma sheath at the center of the confining region, with no EB; (c) Cross section of the EB imaged on a scintillating screen; (d) Measured EB profile (continuous line) along the diameter of the cross section shown in (c) with a FWHM of $4.5 \pm 0.5 \mathrm{~mm}$, fitted with a Gaussian function (dotted line). For reference, the cluster is drawn in yellow in order to indicate its location and diameter relative to the beam cross section and the difference in the intensity of the beam on both sides of the cluster.

near the dust cluster shown from the top in Fig. 2(b). The EB has also been sent on a scintillating $\mathrm{P} 43$ phosphor screen, from which its transverse profile is recorded as shown in Fig. 2(c). In Fig. 2(d) the measured EB profile is fitted with the Gaussian function $\left.2.466 /(2 \pi A) \exp \left[-\left(\xi-8.1 \times 10^{-3}\right)^{2}\right) / 2 A^{2}\right]$, where $A=1.764 \times 10^{-3}$ and $\xi$ is a radial coordinate perpendicular to the EB axis. The full extension of the EB spot as seen in Fig. $2(\mathrm{c})$ is $\approx 9 \mathrm{~mm}$.

\section{OBSERVATION OF CLUSTER ROTATION DYNAMICS}

A stable dust cluster shown in Fig. 3(a) rotates when it is irradiated by the EB, as presented in Figs. 3(b)-3(c). Here the energy of the pulsed EB is $11 \mathrm{keV}$ and its duty cycle is $2.2 \times 10^{-3}$. The dust particles trajectories are tracked in time by using dedicated Fiji (ImageJ) software [33,34]. Typically, the trajectories follow concentric circles as long as the cluster keeps its structure unaltered. There are however circum- 


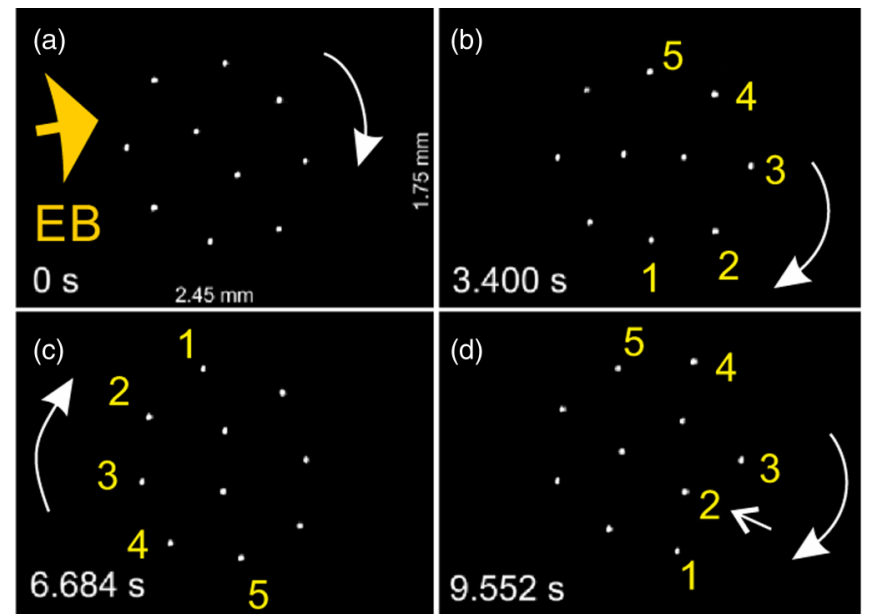

FIG. 3. (a) Cluster rotation in the horizontal plane and direction of the EB at $11 \mathrm{keV}$ : (a) initial position when the EB is turned on; (b) rotation induced by the EB torque; outer dust particles labeled 1 to 5 are tracked in time; (c) rotation with angle $\approx \pi$ around the cluster center, and (d) itinerancy towards the cluster center of dust particle 2 .

stances when the trajectories deviate from a circular path, as shown in the image of Fig. 3(d). Here the outer dust particles are strongly pushed by the EB and one of them "slips" inside the cluster and "sticks" to the two inner particles. After a short period of time $(\approx 0.5 \mathrm{~s})$, another inner particle is eventually expelled out and the cluster regains its initial configuration. This "stick-slip" behavior or the temporary loss of crystalline order has been previously linked to phase transitions between crystalline and "liquid" states [14,35,36].

We can identify three types of dynamics by varying the parameters of the EB: (1) at lower energy $\sim 8-9 \mathrm{keV}$ and implicitly low beam current $\approx 0.2-0.4 \mathrm{~mA}$ the stable cluster rotates as a whole; (2) at $10-12 \mathrm{keV}$ and $0.6-1 \mathrm{~mA}$ the motion of the inner dust particles becomes for periods of times decoupled from the rotation of the outer particles similar to intershell rotation [19] and particle itinerancy inside the cluster arises; and (3) at $\gtrsim 13 \mathrm{keV}$ and larger beam currents $\gtrsim 1.2 \mathrm{~mA}$ the crystal arrangement breaks down and no coherent rotation can be observed.

In order to investigate the dynamics of the cluster we employ an approach based on studying the rotational features of the trajectories [37]. We introduce the horizontal and vertical coordinates in the images of Fig. $3, \hat{x}$ and $\hat{y}$, respectively. The center of rotation is chosen at the center of the cluster $\left(x_{C}, y_{C}\right)$, as shown in Fig. 4(a). We introduce the angle $\phi_{i}(t)=$ $\arctan \left[\left(y_{i}(t)-y_{C}\right) /\left(x_{i}(t)-x_{C}\right)\right]$ spanned by the trajectory of each dust particle $\mathrm{i}$ with planar coordinates $x_{i}(t), y_{i}(t)$. The angular speed of a dust particle is $\omega_{i}=d \phi_{i}(t) / d t$, while its speed is $v_{i}=\omega_{i} R_{i}$, where $R_{i}$ is the radius from the rotation center. In Fig. 4(a) the outer dust particles follow steady circular trajectories with a radius $R_{i} \approx 0.5 \mathrm{~mm}$, while the two inner dust particles move on a circle with a smaller radius $\approx 0.2-0.3 \mathrm{~mm}$. We observe occasionally itinerancy between the trajectories defined by these two radii, corresponding to a change of crystalline arrangement [30] as shown in Fig. 3(d).

The unwrapped angle of each dust particle increases in time with $2 \pi$ after each full rotation, as shown in Fig. 4(a). For
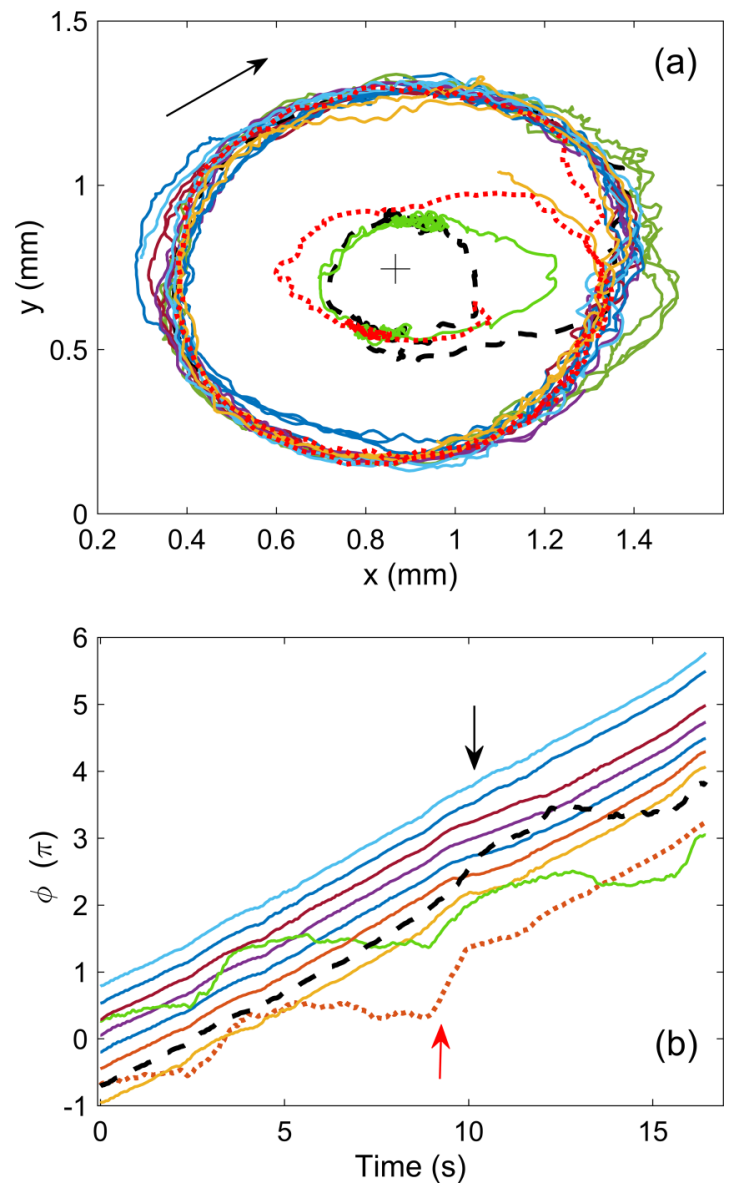

FIG. 4. (a) Measured dust trajectories induced by the pulsed EB with energy $11 \mathrm{keV}$ around the center of rotation (marked with + ); (b) Evolution in time of the unwrapped rotational angle $\phi$ of each dust particle. Dust particles itinerancy between inner and outer trajectories (with dashed and dotted lines) at $t \approx 9.5 \mathrm{~s}$ is shown with arrows.

the outer dust particles the angle varies linear in time with the average angular speed given by the slope of $\phi_{i}(t)$ as in Fig. 4 (b). For the inner dust particles we notice that $\phi_{i}(t)$ increases irregularly in time. The itinerancy of a dust particle inside the cluster can be easily detected by inspecting the shape of the curve $\phi_{i}(t)$. This occurs at well defined moments as marked by the arrows in Fig. 4(b), where the dashed curve shows the particle that slips inside the cluster, while the dotted curve shows the particle that is expelled out to the edge. In fact, from the inflection of the curves $\phi_{i}(t)$ we can infer the time interval when three particles are found inside the cluster, $\Delta t \approx 0.5 \mathrm{~s}$, i.e. from 9.5 to $10 \mathrm{~s}$.

The itinerancy as observed in our experiment is the result of two mechanisms: the EB strongly pushing on the dust particles and the action of the repulsive "circular wall" of the potential well in which the cluster resides. The dust particles facing directly the EB are pushed strongly and spin fast catching up the other dust particles of the cluster. They move circularly, contained by the repulsive "wall" of the sheath field. A dust particle can then deviate radially into the cluster and slows down as it gains an inner position. Had the confining potential been weaker the particles would flow straight, 
along the direction of the EB. At the same time the EB forcing is weak and does not exert a too strong pushing force on the dust particles which could overcome the potential barrier of the confining sheath field.

\section{SIMULATIONS OF CLUSTER ROTATION}

The rotational motion of the dust cluster interacting with a continuous EB (as opposed to the pulsed beam in the experiment which has also a higher energy) has been simulated with the YOAKUM code, a molecular dynamics simulation tool derived from the DEMON code [38]. A system of 10 dust particles with the same size, density and charge as in the experiment is established by distributing them randomly over a $10 \times 10 \mathrm{~mm}$ grid, each with an arbitrary velocity between $\pm 1 \mathrm{~mm} \mathrm{~s}^{-1}$. The dust particles are let to self-arrange, while their evolution in time is monitored subject to the forces applied on them, including here the electron drag force $2 \pi r_{d}^{2} n_{e}^{E B} E_{E B}$, where $n_{e}^{E B}$ is the electron density in the EB, and $E_{E B}$ is the beam energy [12]. The formula is in agreement with derivations of the EB current and force in other works $[9,10,39,40]$. The electron drag force is deduced from the momentum integral following $[41,42]$ and the orbital-motion-limited (OML) theory of dust charging [43,44]: $\mathbf{F}_{\mathbf{e}}=m_{e} \int \vec{v} f_{e}(\vec{v}) \sigma_{e}^{t r}(v) v d^{3} v$, where $m_{e}$ is the electron mass, $\vec{v}$ is the electron speed, $f_{e}(\vec{v})$ is the electron speed distribution function and $\sigma_{e}^{t r}=\sigma_{e}^{\text {coll }}+\sigma_{e}^{\text {orb }}$ is the total momentum transfer cross section by direct and Coulomb collisions. The electron drag force is the sum of direct collision and Coulomb drag forces $F_{e}=F_{e}^{\text {coll }}+F_{e}^{\text {Coul }}$. The cross section of direct collisions is

$$
\sigma_{e}^{\text {coll }}= \begin{cases}\pi r_{d}^{2}\left(1+\frac{2 e V_{d}}{m_{e} v^{2}}\right), & \text { if } \frac{m_{e} v^{2}}{2} \geqslant e\left|V_{d}\right| \\ 0, & \text { if } \frac{m_{e} v^{2}}{2}<e\left|V_{d}\right|,\end{cases}
$$

where $V_{d}\left(V_{d}<0\right)$ is the electrostatic potential of the dust particle. The electrons will be collected on the dust surface if their kinetic energy is high enough to overcome the potential barrier of the charged dust particle. Here the charge is related to the dust potential in the OML framework, $V_{d}=Q_{d} / 4 \pi \varepsilon_{0} r_{d}$. The second cross section describes the interaction of electrons which pass close enough by a microparticle without being trapped on its surface, and transfer a fraction of their momentum via the Coulomb field:

$$
\sigma_{e}^{\text {orb }}=2 \pi r_{C}^{2} \ln \left(\frac{\lambda_{D}^{2}+r_{C}^{2}}{\rho_{C}^{2}+r_{C}^{2}}\right) .
$$

Here $\lambda_{D}$ is the total screening length $1 / \lambda_{D}^{2}=1 / \lambda_{D e}^{2}+$ $1 / \lambda_{D i}^{2}, \lambda_{D e}^{2}$ and $\lambda_{D i}^{2}$ are the electron and ion Debye lengths, respectively, $r_{C}=e Q_{d} / 4 \pi \varepsilon_{0} m_{e} u_{e}^{2}$ is the Coulomb radius, and $\rho_{C}=r_{d} \sqrt{1-2 r_{C} / r_{d}}$ is the maximum impact parameter. The above equations are simplified by the assumption of a monoenergetic electron beam $E_{E B}=m_{e} u_{e}^{2} / 2$ with distribution function $f_{e}(\vec{v})=n_{e}^{E B} \delta\left(v_{x}-u_{e}\right) \delta\left(v_{y}\right) \delta\left(v_{z}\right)$ and with kinetic energy much higher than the electrostatic potential energy of the dust charge: $e\left|V_{d}\right| \ll m_{e} u_{e}^{2} / 2$. Also we note that since $r_{C} \ll r_{d}$ we obtain $F_{e}^{\text {Coul }} \ll F_{e}^{\text {coll }}$.

Neutral atoms are also subject to collisions with the beam electrons, however, the momentum transferred to neutrals is low. On average the energy lost by an electron after a collision with a neutral atom is a fraction $2 m_{e} / m_{n} \approx 10^{-5}$ of its kinetic energy, where $m_{n}$ is the mass of an $\mathrm{Ar}$ atom [45]. For a $12 \mathrm{keV}$ electron it is of the order of $0.12 \mathrm{eV}$. The total electron elastic-scattering cross section at $12 \mathrm{keV}$ on Ar atoms is $\sigma_{\text {elas }} \sim 2 \times 10^{-21} \mathrm{~m}^{-2}$ [46]. The elastic collision frequency of electrons from the beam is $\approx n_{n} \sigma_{\text {elas }} u_{e}=3.8 \times 10^{8} \mathrm{~s}^{-1}$, where $n_{n}=3.16 \times 10^{21} \mathrm{~m}^{3}$ is the neutral density and $u_{e}=$ $6.4 \times 10^{7} \mathrm{~ms}^{-1}$. The effective number of electron collisions per unit length is low therefore the drag force that could be exerted on the dust particles due to EB-induced neutral flow is negligible.

In the simulations $n_{e}^{E B}=10^{13} \mathrm{~m}^{-3}$ as in Ref. [12], while $E_{E B} \approx 1 \mathrm{keV}$. The ions are at the room temperature. The other forces are: the dust-dust interactions $\propto$ $Q_{d}^{2} r_{D}^{-2} \exp \left(-r_{D} / \lambda_{D}\right)$, where $r_{D}$ is the distance between dust particles and $\lambda_{D} \approx 63.6 \mu \mathrm{m}$, the neutral drag force $-\gamma v$ with $\gamma=1.5 \times 10^{-11} \mathrm{~N} \mathrm{~s} \mathrm{~m}{ }^{-1}$, and a radial confining force $-m_{d} k r$, where $m_{d}=(1.3 \pm 0.1) \times 10^{-12} \mathrm{~kg}$ is the mass of a dust particle, $k=5 \times 10^{3} \mathrm{Nm}^{-1}$ and $r=\sqrt{x^{2}+y^{2}}$. The choice of $k$ allows for a confinement of the cluster at the size observed in the experiment. The equation of motion of each dust particle is solved using a fourth-order Runge-Kutta algorithm using a time step of $10^{-4} \mathrm{~s}$.

Once a stable dust cluster is established, as shown in the inset of Fig. 5(a), an EB propagating along the $\hat{x}$ direction is applied. The EB has a planar spatial profile in the $\hat{y}$ direction and a width $-1 \leqslant y \leqslant 0.25 \mathrm{~mm}$, thus creating the means for a torque acting on the cluster, relative to its center $(x, y=0)$. As opposed to the experiment, here, for simplicity, the transverse profile (i.e., in the $\hat{y}$ direction) of the EB is flat. The strength (or electron density) of the EB is set to decrease exponentially in the Ox direction with a characteristic scale length $\Lambda_{E B}=5 \mathrm{~mm}$. The beam is at full strength at $x=-20 \mathrm{~mm}$ from the cluster center. This results in a drag force attenuation factor $\approx 1.8 \times 10^{-2}$. Also the EB energy is lower by a factor of 10 , i.e., $\sim 1 \mathrm{keV}$ in the simulation vs $\sim 10 \mathrm{keV}$ in the experiment. The total attenuation of the $\mathrm{EB}$ is $1.8 \times 10^{-3}$, obtained by multiplying these two previous factors. This attenuation is needed in order to make up for the regime when the pulsed EB is on and off in the experiment, with duty cycle $D=2.2 \times 10^{-3}$. We emphasize that the EB operation mode (continuous in the simulation vs pulsed in the experiment) is leading to differences in the interaction time seen in Fig. 5, i.e., longer in the simulations than in the experiment and lower EB energy (below $1 \mathrm{keV}$ ) where the torque starts to produce a rotational effect on the cluster. The simulated dust particles trajectories are presented in Fig. 5(a), which can be viewed as analogous of Fig. 4(a). The same rotational motion and "stick-slip" dynamics are observed in the simulations shown in Fig. 5(b). The model is based on the main drag effects exerted by the electrons on the dust particles without accounting for the charge fluctuations arising due to EB charging [47], secondary emission [48], or local gas ionization [49].

\section{TORQUE EVALUATION AND DISCUSSION}

The torque is arising from the difference in the relative intensity of the beam on one side of the cluster relative to the other. Over a distance of $1 \mathrm{~mm}$ near the peak of the Gaussian profile in Fig. 2(d) one can see a large drop in the EB intensity, from 250 to $\approx 170$ arb. units, which creates the torque on the 

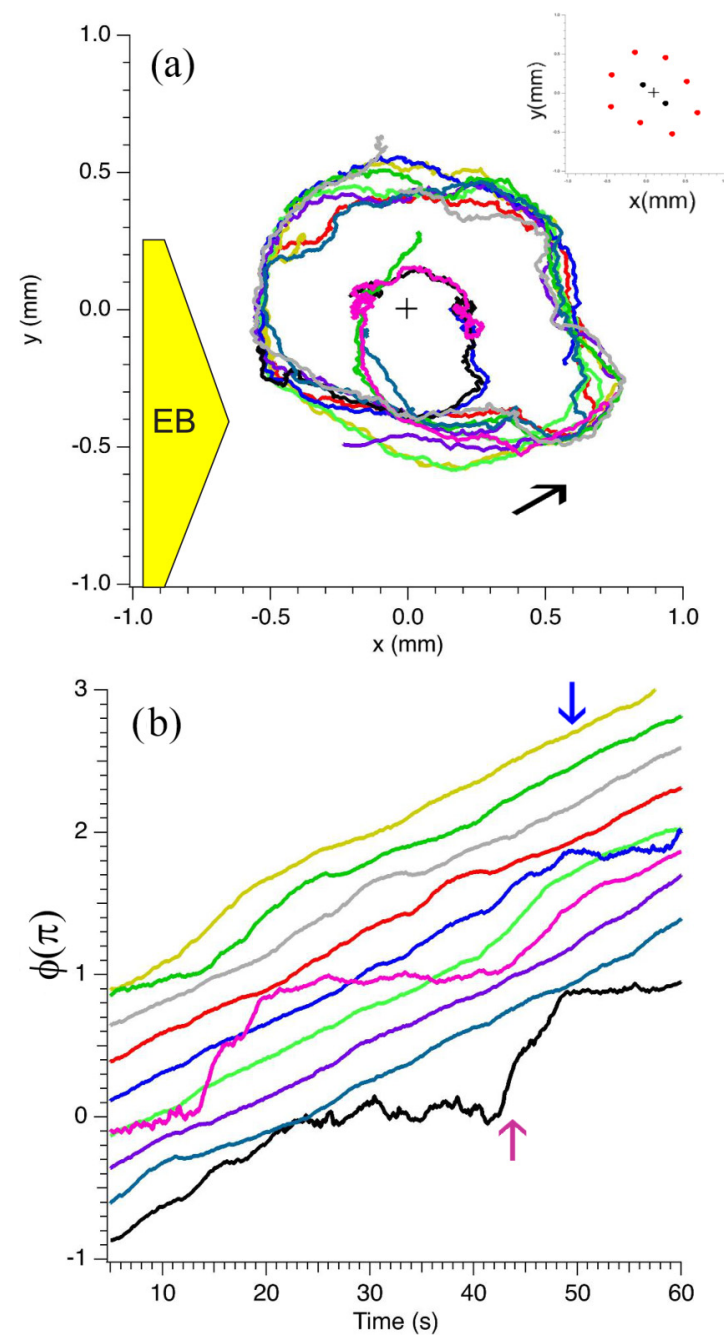

FIG. 5. (a) Simulated dust trajectories for a continuous EB with energy $0.75 \mathrm{keV}$; the inset shows the initial position of the cluster, while the arrow shows the width of the EB. The cluster center is in the origin of axes; (b) Simulated evolution in time of the unwrapped rotational angle $\phi$ of all dust particles. Itinerancy between the inner and outer trajectories (with magenta and blue line) is seen at $t \approx 40 \mathrm{~s}$, marked by arrows.

cluster. The parameters of the experiment (beam size, energy, beam current, cluster size, beam profile, potential well where the cluster is positioned) are all correlated and correspond to the observed phenomena.

We can estimate the total torque $\tau_{E B}$ exerted by the EB from the rotational dynamics of the cluster $I d \omega / d t=\tau_{E B}-$ $\sum_{i=1}^{10} R_{i} \gamma v_{i}$, where $I=\sum_{i=1}^{10} R_{i}^{2} m_{d}$ is the cluster moment of inertia and $\gamma v_{i}$ is the friction force with the neutral gas acting on each dust particle [18]. For steady rotation the EB torque is equilibrated by the friction force torque: $\tau_{E B} \approx \sum_{i=1}^{10} R_{i}^{2} \gamma \omega_{i}$, where $\tau_{i}=R_{i}^{2} \gamma \omega_{i}$ is the torque acting on a single dust particle. For the case of irradiation at $11 \mathrm{keV}$ we estimate $\tau_{E B}=3.1 \pm$ $0.15 \times 10^{-11} \mathrm{Nm}$.

The measured rotation speed is observed to increase with the EB energy, for a stable cluster. The lowest average angular speed is $\omega=1.2 \pm 0.05 \times 10^{-3} \mathrm{rad} \mathrm{s}^{-1}$ at $8 \mathrm{keV}$, as shown in Fig. 6(a). A total torque $\tau_{E B}=4.1 \pm 0.2 \times 10^{-14} \mathrm{Nm}$
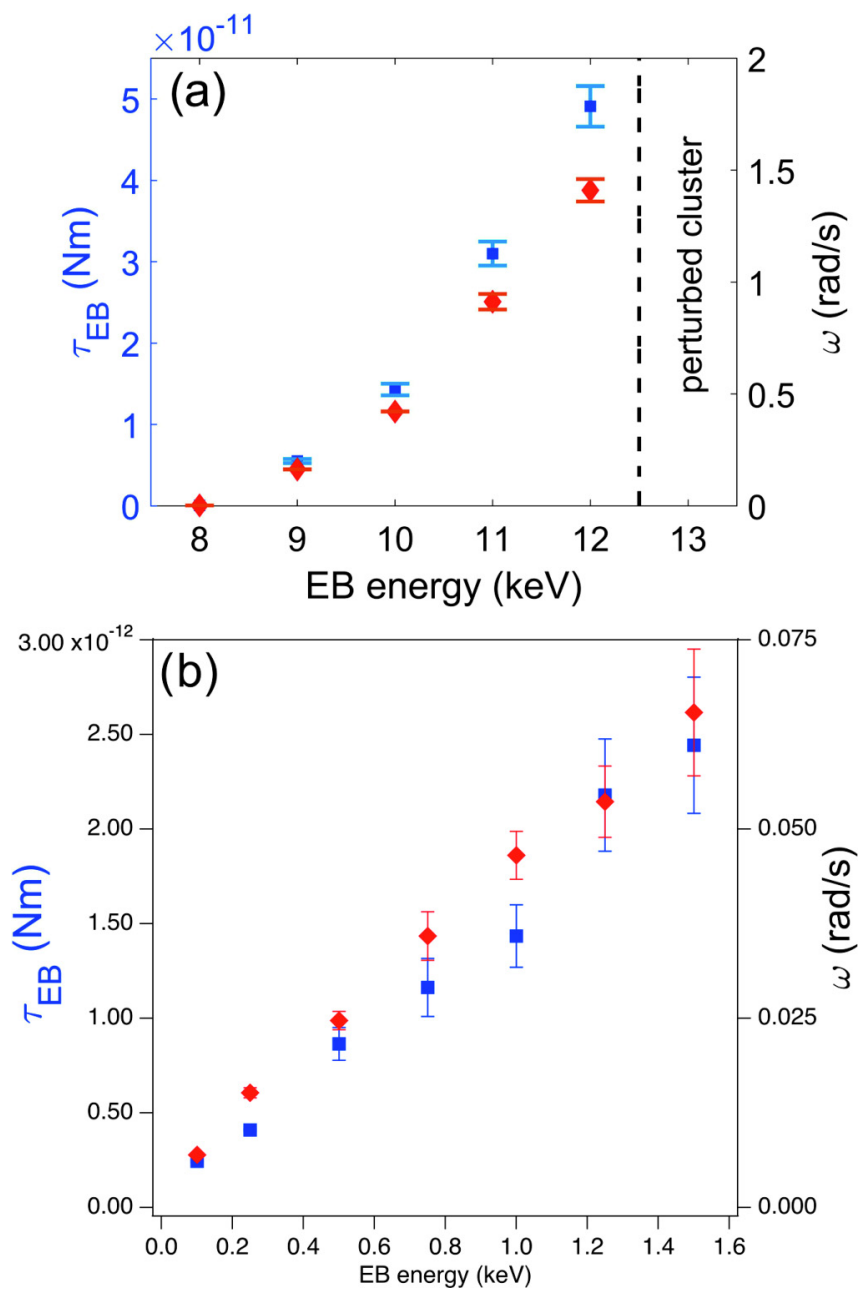

FIG. 6. (a) Measured torque $\tau_{E B}$ (squares) exerted by the pulsed $\mathrm{EB}$ on the whole cluster and average angular speed of rotation $\omega$ (diamonds) of the outer cluster ring; (b) Simulated total torque $\tau_{E B}$ (squares) exerted by the continuous $\mathrm{EB}$ and average angular speed $\tau_{E B}$ (diamonds).

acting on all cluster particles corresponds to this slow, yet well observable, rotation. At $9 \mathrm{keV}, \omega=0.16 \pm 0.006 \mathrm{rad}$ $\mathrm{s}^{-1}$ and increases to $\omega=1.41 \pm 0.05 \mathrm{rad} \mathrm{s}^{-1}$ at $12 \mathrm{keV}$. The same ascending trend is observed for the torque: $\tau_{E B}=$ $5.5 \pm 0.26 \times 10^{-12} \mathrm{Nm}$ to $\tau_{E B}=4.9 \pm 0.25 \times 10^{-11} \mathrm{Nm}$ for these EB energies, respectively. Errors are calculated from the standard deviation (SD) of $\omega_{i}$ and $\tau_{i}$ for each dust particle.

The increase in the rotation speed and torque in Fig. 6(a) with beam energy is not linear as predicted by the electron drag force. This is caused by the variation of the EB current with voltage in the experiment. We observe that the EB current has a tendency to increase at higher voltage and thus the EB has a higher electron density. In the simulation, however, the EB current and electron density in the beam are kept constant and thus the rotation speed and torque increase linearly with the EB energy.

At $11 \mathrm{keV}$ and especially at $12 \mathrm{keV}$ dust oscillations and itinerancy are an indication of a transition to a less stable state of the cluster which for short periods of time changes its structure. This is reflected in the SD which increases as 
the rotation of the outer dust particles is perturbed. In fact at $13 \mathrm{keV}$ all particles move rather chaotically and no rotation of the structure can be observed. The momentum transferred from the EB is converted into turbulent kinetic energy of the whole cluster constituents.

At lower EB energies, below $8 \mathrm{keV}$, the beam is not properly focused for the given magnetic field configuration employed in the experiment. The EB output through the $0.5 \mathrm{~mm}$ hole is dropping significantly and no rotational effect on the cluster is observed. During the experiment the magnetic field strength given by the two focusing coils is kept unchanged.

In separate experiments we could observe rotation in clusters with 5 and 9 particles but also in large crystals with tens of dust particles. It appears that in many observations a higher EB energy ( $\gtrsim 13 \mathrm{keV}$ ) leads inevitably to the destruction of the cluster arrangement.

In the simulations $\omega$ increases relatively smoothly with the EB energy, as shown in Fig. 6(b). At $1 \mathrm{keV}$ the rotation speed $0.045 \mathrm{rads}^{-1}$ and torque $1.75 \times 10^{-12} \mathrm{Nm}$ are lower by a factor of 10 than in the experiment, i.e., $0.42 \mathrm{rads}^{-1}$ and $1.43 \times 10^{-11} \mathrm{Nm}$, respectively. Dust itinerancy becomes more frequent at higher EB energy, as shown by the increased $\mathrm{SD}$ of the average rotation speed $\omega$, leading eventually to a completely perturbed cluster.

The angular speed is smaller in the simulation due to the forces opposed to dust motion, including here the radial confining force $-m_{d} k r$. Our cluster model is a simplification and consists only of negatively charged spheres which repel each other while in the experiment the confinement of the dust particles depends on many more factors, such as the profile of the sheath electric field, the ion flow in the sheath and the distribution of ions and electrons inside the sheath. More generally, it is not uncommon in simulations to vary the strength of the different forces to evidence a particular phenomenon. In Ref. [50] the damping coefficient corresponding to the friction force with the neutral gas atoms is taken smaller by an order of magnitude in the simulation of a dust flow immersed in the plasma sheath, when compared to the experiment. In other studies, the gas pressure and implicitly the friction force with the neutral atoms has been reduced in the simulation with two orders of magnitude compared to the experiment, in order to allow for the propagation of solitary dust waves [51].

\section{CONCLUSIONS}

We demonstrate experimentally the rotation of a millimeter size cluster of charged dust particles levitated in plasma by the torque of an EB with energy in the 8-12 keV range and beam currents lower than $\approx 1 \mathrm{~mA}$. The main force at work is the drag force exerted by the electrons of the beam which collide with the dust particles. The cluster is strongly coupled, confined into a potential well and constrained to move circularly by the EB. Although the round EB spot has a larger diameter compared to the size of the cluster, due to its Gaussian profile in cross section the cluster is irradiated differently on one side closer to the beam axis, than on the opposite side. A direct proof of the action of the electron drag force comes from the increasing rotation speed of the cluster with the EB energy.

A simple simulation model for dust cluster irradiation with an EB exhibits all the peculiarities observed in the experiment, such as cluster rotation, particle itinerancy, and increased rotation speed with beam energy. We emphasize that in the model the electron beam is turned on continuously while in the experiment the beam is pulsed at $56 \mathrm{~Hz}$ with a pulse duration of $40 \mu \mathrm{s}$. The beam profile in the simulation is such that it irradiates only a side of the cluster, thus favoring the creation of a net torque.

These results demonstrate the capability of an EB with energy $\approx 10 \mathrm{keV}$ to rotate and manipulate large microscopic clusters (of order $1 \mathrm{~mm}$ ) immersed in plasma. Moreover, the momentum transfer from the EB to the particles is of relevance for exciting new types of rotational dynamics in microscopic structures, e.g., in micromotors [52].

\section{ACKNOWLEDGMENTS}

D.T. and C.M.T. acknowledge support from the ROSA on project STAR No. 123 (C3-2016), and from the Romanian Ministry of Research and Innovation-MCI on Project No. PN-16-47-01-02. J.D.W acknowledges support from the US National Science Foundation (NSF), Grants No. PHY1615421, and No. PHY-2010122. L.S. and E.T. acknowledge support from NSF Grant No. EPSCoR OIA-1655280. The authors thank the anonymous referees for their valuable comments and suggestions.
[1] A. Ashkin, Acceleration and Trapping of Particles by Radiation Pressure, Phys. Rev. Lett. 24, 156 (1970).

[2] A. Ashkin, Application of laser radiation pressure, Science 210, 1081 (1980).

[3] A. Ashkin and J. M. Dziedzic, Optical trapping and manipulation of viruses and bacteria, Science 235, 1517 (1987).

[4] D. Gao, W. Ding, M. Nieto-Vesperinas, X. Ding, M. Rahman, T. Zhang, C. Lim, and C.-W. Qiu, Optical manipulation from the microscale to the nanoscale: Fundamentals, advances and prospects, Light Sci. Appl. 6, e17039 (2017).

[5] O. M. Marago, P. H. Jones, P. G. Gucciardi, G. Volpe, and A. C. Ferrari, Optical trapping and manipulation of nanostructures, Nat. Nanotechnol. 8, 807 (2013).
[6] C. Su, M. Tripathi, Q.-B. Yan, Z. Wang, Z. Zhang, C. Hofer, H. Wang, L. Basile, G. Su, and M. Dong et al., Engineering singleatom dynamics with electron irradiation, Sci. Adv. 5, eaav2252 (2019)

[7] J. Verbeeck, H. Tian, and G. Van Tendeloo, How to manipulate nanoparticles with an electron beam? Adv. Mater. 25, 1114 (2013).

[8] B. J. Roxworthy, A. M. Bhuiya, X. Yu, E. K. C. Chow, and K. C. Toussaint, Jr., Reconfigurable nanoantennas using electron-beam manipulation, Nat. Commun. 5, 4427 (2014).

[9] L. M. Vasilyak, M. N. Vasil'ev, S. P. Vetchinin, D. N. Polyakov, V. E. Fortov, The action of an electron beam on dust structures in a plasma, J. Exp. Theor. Phys. 96, 440 (2003). 
[10] V. E. Fortov, A. V. Gavrikov, O. F. Petrov, V. S. Sidorov, M. N. Vasiliev, and N. A. Vorona, Superhigh dust charging by high-voltage electron beam, Europhys. Lett. 94, 55001 (2011).

[11] C. M. Ticos, D. Ticos, and J. D. Williams, Kinetic effects in a plasma crystal induced by an external electron beam, Phys. Plasmas 26, 043702 (2019).

[12] C. M. Ticos, D. Ticos, and J. D. Williams, Pushing microscopic matter in plasma with an electron beam, Plasma Phys. Contr. Fusion 62, 025003 (2020).

[13] H. Thomas, G. E. Morfill, V. Demmel, J. Goree, B. Feuerbacher, and D. Mohlmann, Plasma Crystal: Coulomb Crystallization in a Dusty Plasma, Phys. Rev. Lett. 73, 652 (1994).

[14] J. H. Chu and Lin I, Direct Observation of Coulomb Crystals and Liquids in Strongly Coupled rf Dusty Plasmas, Phys. Rev. Lett. 72, 4009 (1994).

[15] V. E. Fortov, A. P. Nefedov, V. M. Torchinsky, V. I. Molotkov, O. F. Petrov, A. A. Samarian, A. M. Lipaev, and A. G. Khrapak, Crystalline structures of strongly coupled dusty plasmas in $\mathrm{dc}$ glow discharge strata, Phys. Lett. A 229, 317 (1997)

[16] W. H. Steel, D. A. Law, B. M. Annaratone, and J. E. Allen, Laser induced motion of micrometre size particles in rf plasma sheaths, in Proceedings of the XXIII International Conference on Phenomena in Ionized Gases (Universite Paul Sabatier, Toulouse, France, 1997), p. 194.

[17] A. Melzer, Laser manipulation of particles in dusty plasmas, Plasma Sources Sci. Technol. 10, 303 (2001).

[18] B. Liu, J. Goree, V. Nosenko, and L. Boufendi, Radiation pressure and gas drag forces on a melamine-formaldehyde microsphere in a dusty plasma, Phys. Plasmas 10, 9 (2003).

[19] M. Klindworth, A. Melzer, and A. Piel, V. A. Schweigert, Laserexcited intershell rotation of finite Coulomb clusters in a dusty plasma, Phys. Rev. B 61, 8404 (2000).

[20] U. Konopka, D. Samsonov, A. V. Ivlev, J. Goree, V. Steinberg, and G. E. Morfill, Rigid and differential plasma crystal rotation induced by magnetic fields, Phys. Rev. E 61, 1890 (2000).

[21] F. M. H. Cheung, N. J. Prior, L. W. Mitchell, A. A. Samarian, and B. W. James, Rotation of Coulomb crystals in a magnetized inductively coupled complex plasma, IEEE Trans. Plasma Sci. 31, 112 (2003).

[22] F. Cheung, A. Samarian, and B. James, The rotation of planar2 to planar-12 dust clusters in an axial magnetic field, New J. Phys. 5, 75 (2003).

[23] L.-J. Hou, Y.-N. Wang, and Z. L. Miskovic, Formation and rotation of two-dimensional Coulomb crystals in magnetized complex plasma, Phys. Plasmas 12, 042104 (2005).

[24] K. Matyash, M. Frohlich, H. Kersten, G. Thieme, R. Schneider, M. Hannemann, and R. Hippler, Rotating dust ring in an RF discharge coupled with a dc-magnetron sputter source. Experiment and simulation, J. Phys. D 37, 2703 (2004).

[25] V. Yu Karasev, E. S. Dzlieva, A. Yu. Ivanov, and A. I. Eikhvald, Rotational motion of dusty structures in glow discharge in longitudinal magnetic field, Phys. Rev. E 74, 066403 (2006).

[26] V. Nosenko, A. V. Ivlev, S. K. Zhdanov, M. Fink, and G. E. Morfill, Rotating electric fields in complex (dusty) plasmas, Phys. Plasmas 16, 083708 (2009).

[27] J. Carstensen, F. O. Greiner, L.-J. Hou, H. Maurer, and A. Piel, Effect of neutral gas motion on the rotation of dust clusters in an axial magnetic field, Phys Plasmas 16, 013702 (2009).
[28] F. Huang, Y.-H. Liu, Z. Y. Chen, L. Wang, and M. F. Ye, Cluster rotation in an unmagnetized dusty plasma, Chin. Phys. Lett. 30, 115201 (2013).

[29] T. B. Mitchell, J. J. Bollinger, W. M. Itano, and D. H. E. Dubin, Stick-Slip Dynamics of a Stressed Ion Crystal, Phys. Rev. Lett. 87, 183001 (2001).

[30] W.-T. Juan, J.-W. Hsu, Z.-H. Huang, Y.-J. Lai, and Lin I, Structures and motions of strongly coupled quasi-2D dust Coulomb clusters in plasmas: From small N to large N, Chinese J. Phys. 37, 184 (1999).

[31] D. Ticos, A. Scurtu, and C. M. Ticos, Setup and diagnostic for plasma crystal irradiation with an electron beam (unpublished).

[32] D. Toader, M. Oane, and C. M. Ticos, Collimated electron beam accelerated at $12 \mathrm{kV}$ from a Penning discharge, Rev. Sci. Instrum. 86, 013301 (2015).

[33] J. Schindelin, I. Arganda-Carreras, E. Frise, V. Kaynig, M. Longair, T. Pietzsch, S. Preibisch, C. Rueden, S. Saalfeld, B. Schmid et al., Fiji: An open-source platform for biologicalimage analysis, Nat. Methods 9, 676 (2012).

[34] J. Y. Tinevez, N. Perry, J. Schindelin, G. M. Hoopes, G. D. Reynolds, E. Laplantine, S. Y. Bednarek, S. L. Shorte, and K. W. Eliceiri, TrackMate: An open and extensible platform for single-particle tracking, Methods 115, 80 (2017).

[35] Y.-S. Su, C. Yang, M.-C. Chen, and Lin I, How does the supercooled dusty plasma liquid relax microscopically after quenching? Plasma Phys. Control. Fusion 54, 124010 (2012).

[36] J. D. Williams, E. Thomas, Jr., L. Couedel, A. V. Ivlev, S. K. Zhdanov, V. Nosenko, H. M. Thomas, and G. E. Morfill, Kinetics of the melting front in two-dimensional plasma crystals: Complementary analysis with the particle image and particle tracking velocimetries, Phys. Rev. E 86, 046401 (2012).

[37] C. M. Ticos, E. Rosa, Jr., W. B. Pardo, J. A. Walkenstein, and M. Monti, Experimental Real-Time Phase Synchronization of a Paced Chaotic Plasma Discharge, Phys. Rev. Lett. 85, 2929 (2000).

[38] R. A. Jefferson, M. Cianciosa, and E. Thomas, Simulations of one- and two-dimensional complex plasmas using a modular, object oriented code, Phys. Plasmas 17, 113704 (2010).

[39] M. N. Vasil'ev, N. A. Vorona, A. V. Gavrikov, O. F. Petrov, V. S. Sidorov, and V. E. Fortov, Anomalously high charging of dispersed particles by $25 \mathrm{keV}$ electron beam, Technol. Phys. Lett. 36, 1143 (2010).

[40] O. F. Petrov, O. S. Vaulina, M. M. Vasiliev, E. A. Lisin, M. I. Myasnikov, S. N. Antipov, V. M. Chepelev, X. G. Koss, Y. Tun, and V. E. Fortov, Coulomb systems of strongly interacting dust particles: Laboratory and microgravity conditions, Optoelectron. Instrument. Proc. 50, 442 (2014).

[41] S. A. Khrapak and G. E. Morfill, Dusty plasmas in a constant electric field: Role of the electron drag force, Phys. Rev. E 69, 066411 (2004).

[42] S. A. Khrapak, Electron and ion thermal forces in complex (dusty) plasmas, Phys. Plasmas 20, 013703 (2013).

[43] R. V. Kennedy and J. E. Allen, The floating potential of spherical probes and dust grains. II: Orbital motion theory, J. Plasma Phys. 69, 485 (2003).

[44] X.-Z. Tang and G. L. Delzanno, Orbital-motion-limited theory of dust charging and plasma response, Phys. Plasmas 21, 123708 (2014). 
[45] M. A. Lieberman and A. J. Lichtenberg, Principles of Plasma Discharges and Material Processing (Wiley, New York, 2005), Chap. 3, Atomic Collisions.

[46] F. Salvat, A. Jablonski, C. J. Powell, and A. Y. Lee, NIST Electron Elastic-Scattering Cross-Section Database, Version 4.0, SRD 64, doi: 10.18434/T4NK50, National Institute of Standards and Technology, Gaithersburg, MD (2016); https://srdata. nist.gov/srd64.

[47] S. I. Kopnin, T. I. Morozova, and S. I. Popel, Electron beam action and high charging of dust particles, IEEE Trans. Plasma Sci. 46, 701 (2018).

[48] J. Pavlu, J. Safrankova, Z. Nemecek, and I. Richterova, Dust Charging in space-related laboratory experiments: A review focused on secondary emission, Contrib. Plasma Phys. 49, 169 (2009).

[49] J. X. Ma, M. Y. Yu, X. P. Liang, J. Zheng, W. D. Liu, and C. X. $\mathrm{Yu}$, Ambipolar diffusion in an inhomogeneous dusty plasma, Phys. Plasmas 9, 1584 (2002).
[50] Y. Bailung, B. Chutia, T. Deka, A. Boruah, S. K. Sharma, S. Kumar, J. Chutia, Y. Nakamura, and H. Bailung, Vortex formation in a strongly coupled dustyplasma flow past an obstacle, Phys. Plasmas 27, 123702 (2020).

[51] M. Schwabe, S. A. Khrapak, S. K. Zhdanov, M. Y. Pustylnik, C. Räth, M. Fink, M. Kretschmer, A. M. Lipaev, V. I. Molotkov, A. S. Schmitz, M. H. Thoma, A. D. Usachev, A. V. Zobnin, G. I. Padalka, V. E. Fortov, O. F. Petrov, and H. M. Thomas, Slowing of acoustic waves in electrorheological and string-fluid complex plasmas, New J. Phys. 22, 083079 (2020).

[52] U. G. Butaite, G. M. Gibson, Y.-L. D. Ho, M. Taverne, J. M. Taylor, and D. B. Phillips, Indirect optical trapping using light driven micro-rotors for reconfigurable hydrodynamic manipulation, Nat. Commun. 10, 1215 (2019). 